The TORCH PMT: a close packing, multi-anode, long life MCP-PMT for Cherenkov applications

This content has been downloaded from IOPscience. Please scroll down to see the full text. 2015 JINST 10 C05003

(http://iopscience.iop.org/1748-0221/10/05/C05003)

View the table of contents for this issue, or go to the journal homepage for more

Download details:

IP Address: 130.209.115.202

This content was downloaded on 10/07/2015 at 10:43

Please note that terms and conditions apply. 


\title{
The TORCH PMT: a close packing, multi-anode, long life MCP-PMT for Cherenkov applications
}

\author{
T.M. Conneely, ${ }^{a, 1}$ M.W.U. van Dijk, ${ }^{b}$ C. D’Ambrosio, ${ }^{c}$ N. Brook, ${ }^{b}$ L. Castillo García, ${ }^{c, d}$ \\ E.N. Cowie, ${ }^{b}$ D. Cussans, ${ }^{b}$ R. Forty, ${ }^{c}$ C. Frei, ${ }^{c}$ R. Gao,${ }^{e}$ T. Gys, ${ }^{c}$ N. Harnew, ${ }^{e}$ \\ J. Howorth, ${ }^{a}$ J. Lapington, ${ }^{a, f}$ J. Milnes, ${ }^{a}$ D. Piedigrossi ${ }^{c}$ and C. Slatter ${ }^{a}$ \\ ${ }^{a}$ Photek LTD,26 Castleham Road, St. Leonards-on-sea, TN38 9NS, U.K. \\ ${ }^{b}$ University of Bristol, HH Wills Physics Laboratory, Tyndall Avenue, Bristol, BS8 1TL, U.K. \\ ${ }^{c}$ European Organization for Nuclear Research (CERN), CH-1211, Geneva, 23, Switzerland \\ ${ }^{d}$ École Polytechnique Fédérale de Lausanne, Route Cantonale, 1015 Lausanne, Switzerland \\ ${ }^{e}$ University of Oxford, Denys Wilkinson Building, Keble Road, Oxford, OX1 3RH, U.K. \\ ${ }^{f}$ Space Research Centre, University of Leicester, University Road, Leicester, LE1 7RH, U.K. \\ E-mail: tom.conneely@photek.co.uk
}

ABSTRACT: Photek (U.K.) and the TORCH collaboration are undertaking a three year development program to produce a novel square MCP-PMT for single photon detection. The TORCH detector aims to provide particle identification in the $2-10 \mathrm{GeV} / \mathrm{c}$ momentum range, using a Time-of-Flight method based on Cherenkov light. It is a stand-alone R\&D project with possible application in $\mathrm{LHCb}$, and has been proposed for the LHCb Upgrade. The Microchannel Plate (MCP) detector will provide a single photon timing accuracy of $40 \mathrm{ps}$, and its development will include the following properties: (i) Long lifetime up to at least $5 \mathrm{C} / \mathrm{cm}^{2}$; (ii) Multi-anode output with a spatial resolution of $6 \mathrm{~mm}$ and $0.4 \mathrm{~mm}$ respectively in the horizontal and vertical directions, incorporating a novel charge-sharing technique; (iii) Close packing on two opposing sides with an active area fill factor of $88 \%$ in the horizontal direction. Results from simulations modelling the MCP detector performance factoring in the pulse height variation from the detector, NINO threshold levels and potential charge sharing techniques that enhance the position resolution beyond the physical pitch of the pixel layout will be discussed. Also, a novel method of coupling the MCP-PMT output pads using Anisotropic Conductive Film (ACF) will be described. This minimises parasitic input capacitance by allowing very close proximity between the frontend electronics and the MCP detector.

KeYwords: Photon detectors for UV, visible and IR photons (vacuum); Cherenkov detectors; Timing detectors

\footnotetext{
${ }^{1}$ Corresponding author.
} 


\section{Contents}

1 Introduction 1

2 Design of TORCH \& detector requirements 1

3 MCP readout anode design $\quad 3$

4 Electronic readout $\quad 4$

5 Detector modelling 5

$\begin{array}{lll}6 & \text { Anisotropic conductive film } & 7\end{array}$

7 Conclusion 9

\section{Introduction}

The operating conditions at $\mathrm{LHCb}$ are foreseen to be extremely challenging, with high multiplicity events of order 100 tracks every $25 \mathrm{~ns}$ bunch crossing (i.e. $40 \mathrm{MHz}$ crossing rate). For the photon detector this will result in single photon event rates of $>10 \mathrm{MHz} / \mathrm{cm}^{2}$ up to $36 \mathrm{MHz} / \mathrm{cm}^{2}$ and integrated extracted charge $>5 \mathrm{C} / \mathrm{cm}^{2}[1,2]$. It has been shown that the use of Atomic Layer Deposition (ALD) technology to coat MCPs with a layer material with an enhanced secondary electron emission can extend the lifetime of MCP based devices with bialkali photocathode beyond $5 \mathrm{C} / \mathrm{cm}^{2}[3]$, providing several years of detector operation.

\section{Design of TORCH \& detector requirements}

In TORCH, the Time-of-Flight (TOF) of a traversing particle is measured using Cherenkov light produced in a quartz plate and propagating to the edges via total internal reflection, where it is projected on the detector plane using a cylindrical focussing block, as shown in figure 1 . To achieve $3 \sigma$ separation of pions and kaons with identical momentum at the proposed location of TORCH, a per-track time resolution of $10-15 \mathrm{ps}$ is required. With the current design of TORCH [4] it is expected that, per incident track, at least 30 photons can be detected. The required single photon resolution is therefore $70 \mathrm{ps}$.

A full simulation of TORCH has been set up in Geant4 [5], and is currently being used to study some more complex topics, such as track multiplicity and photon-track association. A map is shown in figure 2 of where the photons of one particular (repeated) track end up on the detector plane.

In order to reconstruct the path (and therefore timing) of each single photon, the angles with respect to the vertical and horizontal axis need to be measured to a precision of $\sim 1 \mathrm{mrad}$. The photon's horizontal angle is projected along the X coordinate axis in figure 2, and vertical angle 


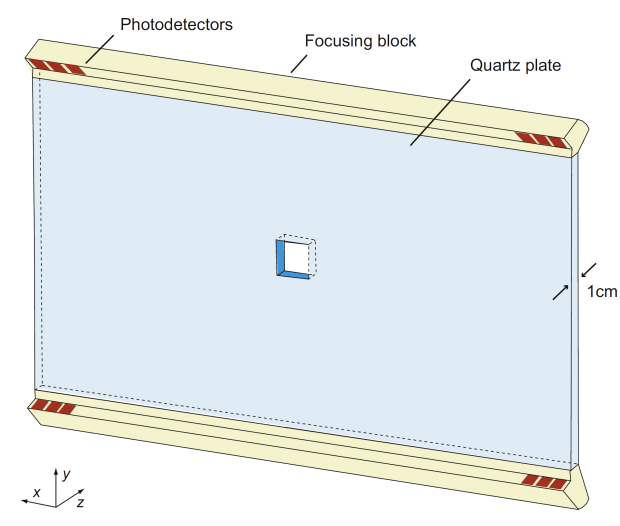

Figure 1 (a). Schematic design of the TORCH $6 \times 5 \mathrm{~m}^{2}$ Cherenkov radiator plate

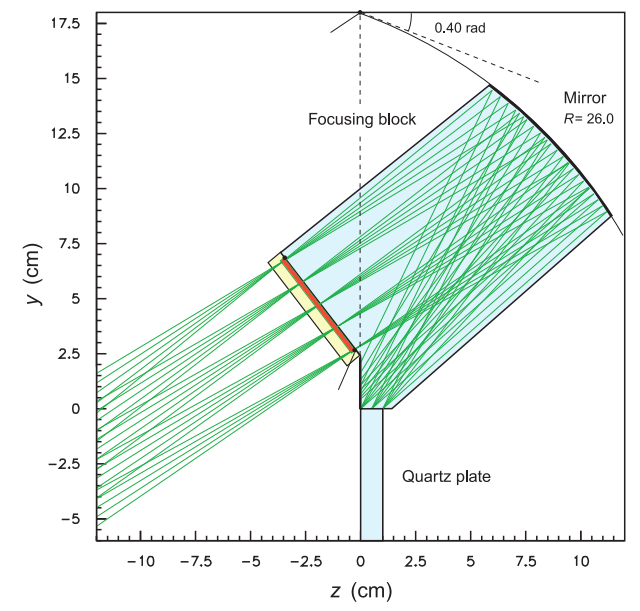

Figure 1 (b). Cross-section of the focussing block [6]

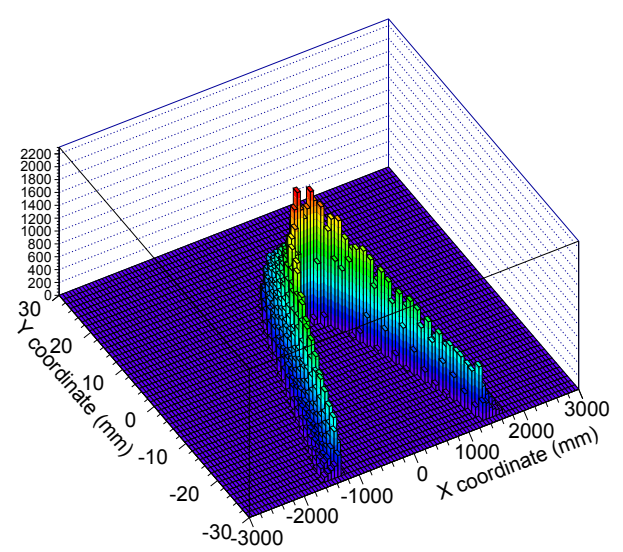

Figure 2. Detector display from GEANT4, showing the sum of photons accumulated from 1000 identical positive kaon tracks passing through the radiator plate, each with a momentum of $10 \mathrm{GeV} / \mathrm{c}$. No detector effects have been accounted for, effectively showing the projection of the Cherenkov cone onto the detector plane.

along the Y coordinate axis on both figure 2 and figure $1 \mathrm{~b}$. Combined with the high resolution timing information of single Cherenkov photons and information from the trackers this allows particle identification.

The photon detectors are arranged in a bank along the edge of the Cherenkov radiator plane, as shown in figure 1a, with a focussing block used to compactly project the photon's vertical angle on to the photodetector's input window. From the expected track and Cherenkov angles at LHCb the angular acceptance of the focussing block has been set to $0.45-0.85 \mathrm{rad}$ covering a range of $0.4 \mathrm{rad}$ projected on to a $60 \times 60 \mathrm{~mm}^{2}$ photodetector input window, with an active area of $53 \times 53 \mathrm{~mm}^{2}$. The angular resolution is given by $\frac{\theta_{r v}}{n_{v} \sqrt{12}}$ where $\theta_{r v}$ is the angular acceptance range for the focussing block ( $0.4 \mathrm{rad})$ and $n_{v}$ is the number of pixels in the vertical dimension. Hence, 128 pixels gives an angular resolution of $0.4 /(128 \sqrt{12})=0.902 \mathrm{mrad}$ meeting the required $1 \mathrm{mrad}$ resolution. 
The horizontal angle is projected along the bank of photo detectors (approximately $6 \mathrm{~m} \mathrm{long),}$ providing a long lever arm for the horizontal angle measurement, and hence a much coarser photon detector pixelisation is acceptable. For a typical event the Cherenkov photons are spread over a $2 \mathrm{~m}$ wide section of the photon detector bank. Therefore the choice for 8 horizontal pixels per $53 \times$ $53 \mathrm{~mm}^{2}$ active area is sufficient to achieve $1 \mathrm{mrad}$ resolution $(6.625 /(2000 \sqrt{12})=0.956 \mathrm{mrad})$.

The choice of $8 \times 128$ pixels per detector leads to a smearing of the intrinsic resolution of the photon propagation time of about $\sim 55 \mathrm{ps}$ [1]. This figure combines the effects of pixelisation and chromatic dispersion on the timing resolution. Subtracting this from the required 70 ps resolution, the remaining components of TORCH (e.g. electronics and intrinsic detector resolution) are limited to a combined time resolution of about 45 ps RMS.

\section{MCP readout anode design}

The TORCH spatial resolution requirement of $8 \times 128$ pixel readout in a $53 \times 53 \mathrm{~mm}^{2}$ square detector could be solved using a multi-anode MCP-PMT, with an anode pitch of $0.414 \mathrm{~mm}$ in the fine direction. Photek has previously developed multi-anode MCP detectors for the IRPICS projects [7], with a pixel pitch of $0.88 \mathrm{~mm}$. These projects used multi-layer ceramic technology to build the readout anode, which creates a monolithic multi-anode readout with excellent vacuum integrity. However, for an $8 \times 128$ multi-anode, the pixel pitch is doubled in one direction, increasing the required electronic connection density and significantly increasing the chance of vacuum leaks during the MCP-PMT manufacturing process.

For TORCH, the proposed solution is to use a structured multi-layer ceramic readout anode, which is a hybrid between a multi-anode detector with direct DC connection from the vacuum envelope to outside of the detector and a charge sharing imaging MCP device. This hybrid anode design capacitively couples the signal from the MCP electron avalanche, collected on a resistive layer inside the vacuum, to anode pads embedded in the ceramic structure. The structure of the hybrid anode is designed to allow for charge sharing between anode pads, allowing the use of charge sharing techniques to increase the detector's spatial resolution beyond the pitch of the anode pads. To perform the charge sharing calculation, a charge measurement at each of the anode's readout pad is needed. The photon's position $X_{\gamma}$ in one dimension can then be naively calculated from

$$
X_{\gamma}=\sum_{i=0}^{n} x_{i} \frac{q_{i}}{Q}
$$

where $x_{i}$ is the spatial coordinate of a readout pad in one dimension, $q_{i}$ is the charge collected by that pad and $Q$ is the total charge collected by all readout pads. An ideal algorithm could use weighting of the charge collection efficiency to further improve imaging resolution.

Additionally, this hybrid anode design has advantages for manufacturing the device and during operation:

- In multi-anode detectors, without capacitive coupling (a challenge for high density readouts), the readout anode must be operated at ground. Hence, to provide the required voltage for MCP operation the input window/photocathode must be operated at high voltage $(3-5 \mathrm{kV})$. This makes coupling the input window of the MCP detector to the TORCH detector's quartz 
focussing block difficult. For the hybrid readout, the AC coupling is an inherent feature of the design, hence the input window can be operated at ground potential with the resistive layer on the anode at high voltage.

- As there are no direct vacuum feed-throughs the chance of introducing a leak should be significantly reduced, increasing yield and hence reducing cost of the device.

- Using a charge sharing technique reduces the number of required readout anodes, reducing the required number of electronic readout channels.

The detector in development for TORCH uses a structured $8 \times 64$ structured multi-anode readout, chosen to meet detector occupancy requirements, which at a detected photon rate of $36 \mathrm{MHz} / \mathrm{cm}^{2}$ requires that no more than three neighbouring readout pads can be used for the charge sharing calculation. This choice avoids pulse pile up, where neighbouring events overlap on the readout anode compromising cluster identification and the imaging algorithm which will see only one event with a position at the centre of gravity of the two events. At $36 \mathrm{Mhz} / \mathrm{cm}^{2}$, the average event rate for a single pad is $2 \mathrm{MHz}$, hence for groups of three pads the event rate is $6 \mathrm{MHz}$. The rate of coincidence for two neighbouring groups, assuming a $25 \mathrm{~ns}$ processing time (restricted by pulse width of NINO output) is then $900 \mathrm{KHz}$ and $2.5 \%$ of events will have closely neighbouring events. It is expected that close neighbour events can still be distinguished in post processing by identifying asymmetrically distributed charge footprints. The MCP detector's hybrid anode has been designed as a $64 \times 64$ device, with an external printed circuit board to connect eight neighbouring pads in the coarse resolution dimension to form a single channel.

\section{Electronic readout}

An important factor in the detector spatial resolution is the choice of readout electronics. For the TORCH project the current solution is a combination of the NINO charge amplifier/discriminator ASIC [8] and HPTDC time-to-digital converter [9].

The NINO ASIC integrates a $\sim 1$ ns rise time amplifier with a time-over-threshold discriminator capable of running at event rates exceeding $10 \mathrm{MHz}$ per channel. Originally developed as an 8 channel device, a 32 channel version has been developed with improved power consumption ( $25 \mathrm{~mW}$ per channel) and optional integrated bias circuitry for LVDS in a $7.5 \mathrm{~mm} \times 3.5 \mathrm{~mm}$ silicon die. The NINO output is a LVDS logic signal with a width related to the collected charge. The pulse width information is intended to correct for amplitude walk of the NINO's discriminator stage to provide timing resolution $<25 \mathrm{ps}$ RMS. For the TORCH application it is intended to use the pulse width as a charge measurement for the charge sharing calculation.

The HPTDC (High Performance Time to Digital Converter) provides a flexible time digitisation solution, with the option of 32 channels operating with 100 ps time bins and 34 ps RMS jitter; or 8 channels with 25 ps time bins and 17 ps RMS jitter using RC-delay line interpolation between groups of four channels.

Previously it has been demonstrated using the HiContent multi-anode MCP-PMT using a NINO and HPTDC for readout, it is possible to achieve $\sim 43$ ps RMS timing resolution [10], after performing amplitude walk correction for the NINO's discriminator stage. Combined in quadrature 
with the 55 ps timing smear from optical/pixellisation effects the full detector system, including the Cherenkov radiator plate, could achieve a single photon timing accuracy of $\sim 70 \mathrm{ps}$.

\section{Detector modelling}

The performance of a detector using a charge sharing technique for interpolating between physical readout pads is impacted by several factors from the MCP detector design, particularly the exact geometry of the hybrid anode and performance of the readout electronics. Hence, a Monte Carlo simulation of the detector and readout electronics has been developed to (i) optimise the structured multi-anode geometry, tuning the charge sharing footprint to maximise position resolution and meet occupancy requirements, (ii) investigate the impact of detector gain, and broad pulse height distribution of the MCP gain process, and (iii) investigate the impact of electronic noise, timing jitter and threshold on position resolution and detector efficiency.

The first stage of the simulation was to model the response of the structured anode to the charge output of the MCP. This was performed in Ansys Maxwell, ${ }^{1}$ by producing a 3D model of the detector/anode structure and placing a test charge on the internal surface of the anode to represent the collected charge from the MCP. The capacitive coupling from the test charge at a position $(x, y)$ to each element was solved for, allowing the fraction of charge collected by each readout pad for a photon at the position $(x, y)$ to be calculated. By moving the test charge across the anode surface, a map of charge collected by each readout pad was built up. To fill the gaps between test charge positions where charge coupling was actually solved for, a B-spline curve was fitted and used to interpolate between data points.

This data was fed into a Monte Carlo model, which begins by modelling the MCP's current output pulse shape and gain followed by a simulation of the electronics response for photon positions generated with a uniform random distribution. For each photon, an MCP gain value was generated from a distribution fitted to a measured MCP pulse height distribution with an identical pore size for the current TORCH MCP detector design. Then using the results of the Ansys Maxwell modelling, the charge collected by each readout pad was calculated.

For each readout pad a current pulse was modelled using an analytical function fitted to a MCPPMT pulse measured using a Agilent $18 \mathrm{GHz}$ sampling oscilloscope. ${ }^{2}$ The fitting function chosen was a Gaussian distribution with a time dependent standard deviation to introduce an asymmetry typical of MCP-PMT pulses with a fast rise time of the order of $100 \mathrm{ps}$ and a slower fall time, and is defined as

$$
\begin{aligned}
& I(t)=-A e^{\frac{\left(t-t_{0}\right)^{2}}{g(t)^{2}}} \\
& g(t)=\frac{2 g_{0}}{1+e^{a\left(t-t_{0}\right)}}
\end{aligned}
$$

where $A$ is the pulse amplitude, $t_{0}$ is the pulse peaking time, $g_{0}$ is the pulse's FWHM and $a$ determines the asymmetry of the pulse's rise time/fall time. Figure 3 shows the measured MCPPMT pulse and corresponding fit which closely models the rise time, fall time and FWHM. The

\footnotetext{
${ }^{1}$ http://www.ansys.com/.

${ }^{2}$ Agilent Infiniium DCA 86100C.
} 


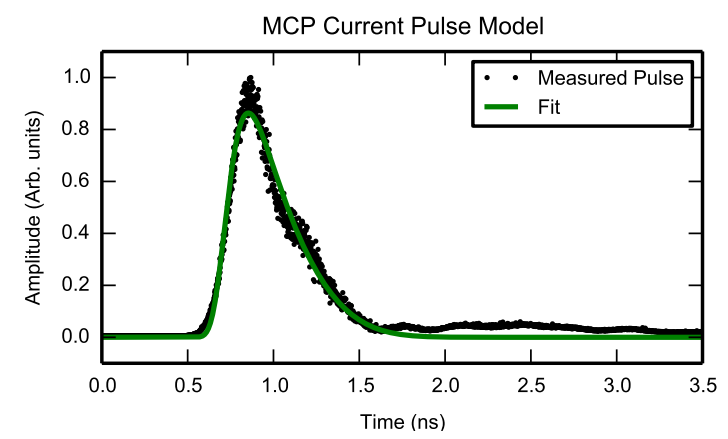

Figure 3. Measured MCP-PMT detector current pulse, with a least square fit of equation 5.1 used in modelling the charge output of the MCP for the readout anode's charge sharing simulation. Values used in the simulation were $g_{0}=0.220 \mathrm{~ns}, a=-3.768 \mathrm{~ns}^{-1}, t_{0}=0.853 \mathrm{~ns}$ and $A$ varied according to MCP gain for each simulated photon.

amplitude of this pulse was then varied according to the MCP gain chosen for each generated photon, so that the integrated charge of the pulse matched the chosen gain value.

The generated detector pulse for each readout pad was passed through a model of the electronics readout. This modelled the amplifier as a low-pass filter with $1 \mathrm{~ns}$ rise time and measured the pulse's time over threshold (TOT), to produce an output matched against calibration data from a sample 32 channel NINO. The leading edge time/pulse width was measured and digitised into 25 ps bins to simulate the HPTDC timing format and dynamic range.

The simulated NINO's output pulse width was converted to signal charge using a previously generated charge calibration lookup table and the simulated charge for each readout pad fed into equation 3.1 to calculate the simulated photon position. Hence, by subtracting the initial generated photon's positions from the reconstructed position, the spatial resolution can be studied. The detector occupancy can be studied as the charge collected by each readout anode is known.

The TORCH MCP-PMT must achieve a spatial resolution equivalent to $8 \times 128$ pixels in a $53 \times 53 \mathrm{~mm}^{2}$ active area, equivalent to a pixel pitch of $0.414 \mathrm{~mm}$ in the fine direction. Hence the charge sharing resolution of the $8 \times 64$ layout, with a pitch of $0.828 \mathrm{~mm}$, must provide a spatial resolution in excess of this value. Figure 4 shows a typical position error distribution (generated photon position subtracted from simulated photon position), with two main contributions (i) a large central peak where more than one pad collected charge above threshold allowing a full charge sharing calculation (ii) broad tails where a single pad fired, hence no charge sharing calculation could be performed. In this case, with $10^{6} \mathrm{MCP}$ gain and a $50 \mathrm{fC}$ threshold, the mean number of pads above threshold per event is 1.36 , meaning there is a significant contribution from single pad events. The position resolution is taken to be the standard deviation of this distribution, which in figure 4 is $0.19 \mathrm{~mm}$. Hence, a $0.414 \mathrm{~mm}$ equivalent pixel can be reconstructed to a confidence level greater than $2 \sigma$. Using a more sophisticated imaging algorithm could further improve this resolution.

This position error was simulated by varying the detector gain and NINO threshold; these are the critical factors in determining SNR due to the low gains required for the high rate operation. Figure 5a shows the simulated position error standard deviation varying these two parameters, indicating for efficient charge sharing resolution a gain $>10^{6}$ is required. 


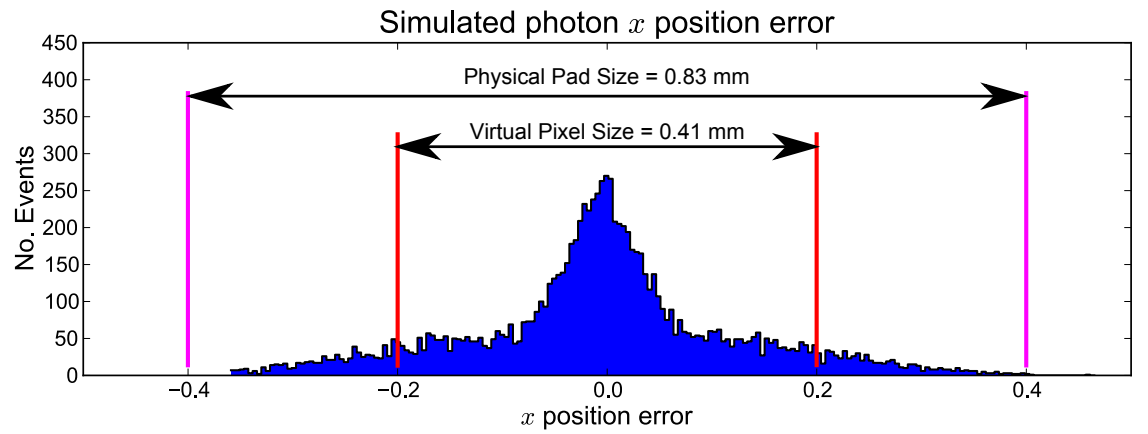

Figure 4. Simulated position error, for $10^{6}$ electron gain and $50 \mathrm{fC}$ NINO threshold. This plot shows the difference between the generated photon's position, and the reconstructed position after detector/electronics simulation. At this position and gain the standard deviation of the distribution is $0.19 \mathrm{~mm}$, allowing reconstruction of a $0.38 \mathrm{~mm}$ pixel to a $2 \sigma$ confidence level.

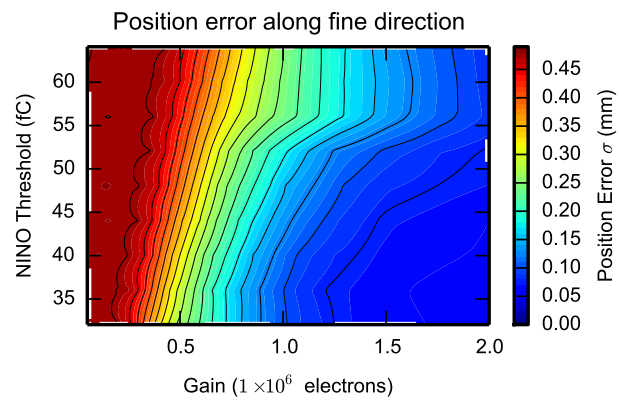

Figure 5 (a). Standard deviation of position error (as plotted in figure 4), showing gains $\geq 10^{6}$ required for achieving desired spatial resolution.

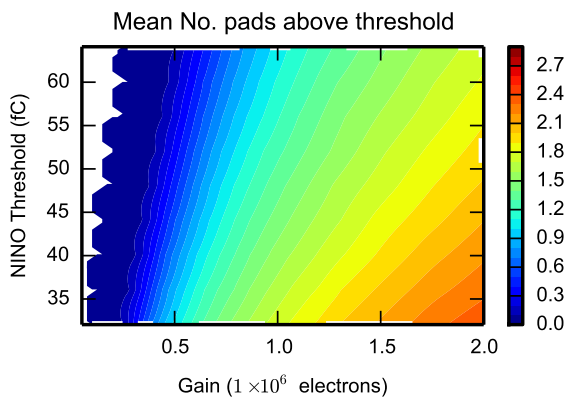

Figure 5 (b). Mean number of pads above threshold, showing that the degree of charge sharing between pads will not impede the desired detector operating rate.

Figure 5. Monte Carlo simulation results as a function of NINO threshold and mean MCP gain.

To study detector occupancy, the number of readout pads with events above the NINO threshold was used as a metric, at $36 \mathrm{MHz} / \mathrm{cm}^{2}$ this was required to be $<3$ for each event. As shown in figure $5 \mathrm{~b}$, this criterion is comfortably met for all gains. Both of these plots were used to optimise the MCP-PMT's anode structure, as there is a trade off in the charge sharing measurement, especially at low gains. This occurs since the charge collected by each individual pad is reduced when charge sharing between pads is increased (i.e. the charge is spread over a larger area). Hence, the SNR for each pads charge measurement is decreased.

\section{Anisotropic conductive film}

For the high density connection to the readout electronics we have chosen to use Anisotropic Conductive Film technology. This is a thin elastomer film $(\sim 100 \mu \mathrm{m}$ thick $)$, with embedded wires at a fixed pitch, which when compressed between two surfaces forms a conductive connection in one direction (through the film thickness). The use of ACF technology allows high density inter- 
connects, and a low capacitive load for the NINO pre-amplifier input compared to using pins as contacts on the detector.

For the TORCH detector we have chosen an ACF film manufactured by ShinEtsu Polymer (MT-P type) [11] which has uniformly distributed conductive wires at $100 \mu \mathrm{m}$ pitch. To form a reliable connection each contact between the detector and interface PCB requires 10 wires, hence the minimum contact pad area is $0.1 \mathrm{~mm}^{2}$. The TORCH detector has $0.45 \times 0.45 \mathrm{~mm}^{2}\left(0.2025 \mathrm{~mm}^{2}\right)$ contact pads on the readout anode, with the PCB connecting groups of 8 along one direction to form the required 8 column readout. Each individual contact pad on the detector exceeds the required contact area, and provides 16 wire contacts per pad, exceeding manufacturer requirements.

In order to prove the use of ACF for TORCH, a $32 \times 32$ multi-anode detector, developed for the IRPICS project (Information Rich Imaging of Cells) [7], was used as a test bed. This detector has external readout contacts, with $0.88 \mathrm{~mm}$ pitch and each gold plated contact is $0.2 \mathrm{~mm}^{2}$, closely resembling the TORCH detector layout $(0.828 \mathrm{~mm}$ pitch in fine direction). Initially the IRPICS detector was illuminated using a pulsed laser, with a 40 ps FWHM (Photek LPG-650) attenuated to single photon levels using ND filters. A test PCB was designed, routing a single pixel to a $50 \Omega$ transmission line connected to a LeCroy $5 \mathrm{GHz} 20 \mathrm{GS} / \mathrm{s}$ oscilloscope, ${ }^{3}$ grounding all other anode connections. The measured detector pulse, shown in figure 6a, closely matches the typical pulse from a single channel MCP-PMT. This measurement was limited by the oscilloscope's bandwidth, so the IRPICS detector's true risetime is faster than shown.

This was followed by measuring crosstalk by scanning a focussed laser across two neighbouring pads: i) in single photon mode using Photek's IPD charge sensitive amplifier (500 ns shaping time) and counting electronics, ii) in single photon mode using the NINO ASIC directly coupled to the IRPICS with counting electronics monitoring detected photon rate. For each crosstalk measurement the LPG-650 laser was focussed down to a spot with $0.2 \mathrm{~mm}$ FWHM measured with an IPD camera (single photon imaging detector), and a translation stage used to step the spot across two neighbouring pads in $0.1 \mathrm{~mm}$ increments.

Each set of IPD electronics thresholds were matched, and set to the valley in the MCP detector's pulse height distribution. At each laser position the detected photon events were counted for a fixed integration period of 180 seconds on each pad, and is shown in figure 6b. Each anode shows a flat pedestal when the laser is centred over that anode, approximately $0.7 \mathrm{~mm}$ wide which compares well with the IRPICS detector's $0.78 \mathrm{~mm}$ anode size, and $0.88 \mathrm{~mm}$ pitch. There is then a steep fall over a range of approximately $0.2 \mathrm{~mm}$ at each anode's edge. These results have not been corrected for the laser spot size $(0.2 \mathrm{~mm}$ FWHM).

This test was then repeated, replacing the two sets of IPD electronics with the NINO amplifier/discriminator as the readout device. The NINO board used, developed by the University of Leicester, is a 64-channel design using two 32-channel NINO ASICs, each chip is supplied with an independent DAC controlled threshold which was set to the same level for both chips. Of the two neighbouring readout anodes, a single channel on separate NINO chips was used. Again the detector's count rate was monitored as a function of laser position. Figure $6 \mathrm{~b}$ shows the crosstalk measured using NINO overlaid on the previously measurement using the IPD electronics, the crosstalk distribution for each anode is similar to the previous results especially for channel 1 . The slight

\footnotetext{
${ }^{3}$ LeCroy Wavemaster 8500A.
} 

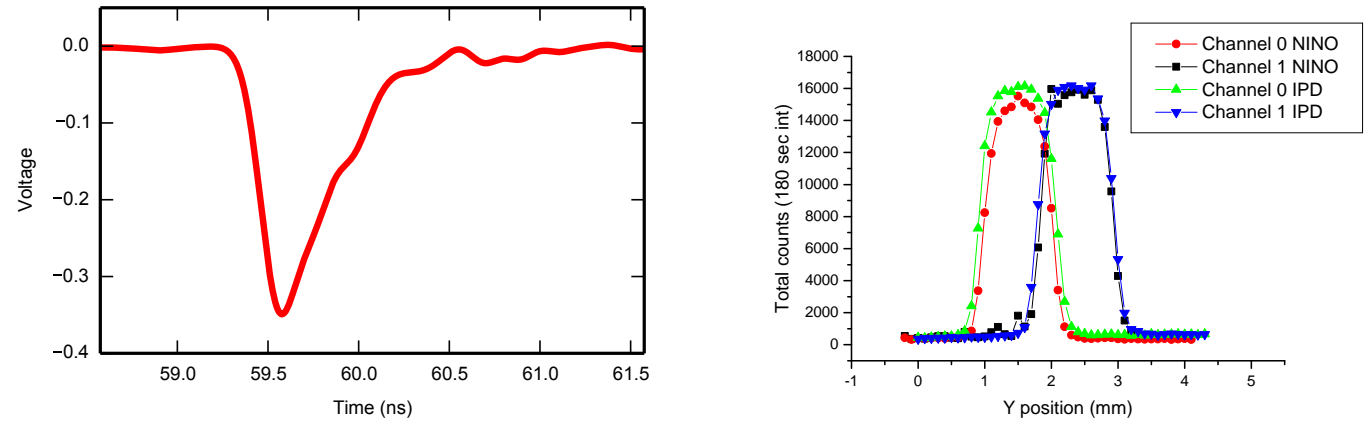

Figure 6 (a). Measured single photon pulse of IR- Figure 6 (b). Crosstalk for IRPICS multi-anode PICS multi-anode MCP-PMT using ACF for cou- MCP detector in single photon counting regime, pling the detector to a readout $\mathrm{PCB}$, closely matching using ACF film to couple detector to readout the typical pulse shape for single channel MCP-PMT PCB.

detectors.

deviation seen on channel 0 from the shape seen on channel 1 is likely due to the thresholds of the two NINO chips not being perfectly matched.

\section{Conclusion}

The TORCH project presents a number of challenges for designing a suitable photon detector for their application, requiring long lifetime $\left(5 \mathrm{C} / \mathrm{cm}^{2}\right.$ extracted charge over detector lifetime), high rate operation $\left(36 \mathrm{MHz} / \mathrm{cm}^{2}\right.$ detected photon), high time resolution $(<50 \mathrm{ps})$ and a high channel count/density readout in a square form factor for minimum dead area.

A novel readout scheme for MCP based detectors has been designed and simulated, which combines the advantages of a multi-anode readout (e.g. highly parallel event readout) with those of a AC coupled, charge sharing design (e.g. decoupling the readout anode from the detector's vacuum envelope). The chosen design successfully meets the TORCH spatial resolution requirements of $8 \times 128$, and provides a sufficient parallel event readout to meet the high detected photon rate expected at TORCH.

We have also demonstrated the viability of anisotropic conductive film as a method of interfacing high density multi-anode MCP detectors to a suitable readout PCB, using the IRPICS direct multi-anode detector. It was shown that the high bandwidth MCP signals are coupled from the detector to readout electronics as expected and that crosstalk between neighbouring anodes of the IRPICs detector is not excessive.

Prototypes of the MCP detector design have been manufactured, implementing a quarter of the anticipated final design's active area. Work will now focus on proving and characterising the MCP detector's performance experimentally with the NINO/HPTDC readout electronics and beam tests have been planned to prove the TORCH concept in conjunction with the MCP detector design. 


\section{Acknowledgments}

The support of the European Research Council is gratefully acknowledged in the funding of the PMT development through an Advanced Grant under the Seventh Framework Programme (FP7) (ERC-2011-AdG 299175-TORCH).

\section{References}

[1] R. Forty, The TORCH project, 2014 JINST 9 C04024.

[2] T. Gys, L. Castillo García, J. Fopma, R. Forty, C. Frei et al., Performance and lifetime of micro-channel plate tubes for the TORCH detector, Nucl. Instrum. Meth. A 766 (2014) 171.

[3] T.M. Conneely, J.S. Milnes and J. Howorth, Extended lifetime MCP-PMTs: Characterisation and lifetime measurements of ALD coated microchannel plates, in a sealed photomultiplier tube, Nucl. Instrum. Meth. A 732 (2013) 388.

[4] M.W.U. van Dijk, N.H. Brook, L. Castillo García, E.N. Cowie, D. Cussans et al., TORCH - $a$ Cherenkov based time-of-flight detector, Nucl. Instrum. Meth. A 766 (2014) 118.

[5] GEANT4 collaboration, S. Agostinelli et al., GEANT4: A Simulation toolkit, Nucl. Instrum. Meth. A 506 (2003) 250.

[6] M. Charles and R. Forty, TORCH: time of flight identification with Cherenkov radiation, Nucl. Instrum. Meth. A 639 (2011) 173 [arXiv: 1009.3793].

[7] J. Lapington, T. Ashton, D. Ross, and T. Conneely, Progress towards a 256 channel multi-anode microchannel plate photomultiplier system with picosecond timing, Nucl. Instrum. Meth. A 695 (2012) 78.

[8] F. Anghinolfi, P. Jarron, A.N. Martemyanov, E. Usenko, H. Wenninger et al., NINO: an ultra-fast and low-power front-end amplifier/discriminator ASIC designed for the multigap resistive plate chamber, Nucl. Instrum. Meth. A 533 (2004) 183.

[9] M. Mota, J. Christiansen, S. Debieux, V. Ryjov, P. Moreira, and A. Marchioro, A flexible multi-channel high-resolution time-to-digital converter asic, IEEE Nucl. Sci. Symp. Conf. Rec. 2 (2000) 9/155.

[10] J. Lapington and T. Conneely, Multi-channel picosecond photon timing with microchannel plate detectors, Nucl. Instrum. Meth. A 648 (2011) S186.

[11] ShinEtsu Polymer, MT-type of inter-connector, http://www.shinpoly.com/products/interconnectors/mt01.shtml, webpage accessed April 2015. 